2. - Differential systems with general boundary conditions, Seminar Reports in Mathematics, University of California Publications in Mathematics 2 (1944), $45-61$.

3. R. H. Cole, General boundary conditions for an ordinary linear differential system, Trans. Amer. Math. Soc. 111 (1964), 521-550.

4. W. M. Whyburn, On related difference and differential systems, Amer. J. Math. 51 (1929), 265-286.

The Virginia Military Institute

\title{
THE ASYMPTOTIC BEHAVIOR OF A CLASS OF NONLINEAR DIFFERENTIAL EQUATIONS ${ }^{1}$
}

\section{DONALD S. COHEN}

We shall study the asymptotic behavior for $t \rightarrow \infty$ of solutions of the following nonlinear differential equation:

$$
u^{\prime \prime}+f(t, u)=0 \text {. }
$$

We suppose that $f(t, u)$ satisfies the following conditions:

$\mathrm{H}-1: f(t, u)$ is continuous in $D: t \geqq 0,-\infty<u<\infty$.

H-2: The derivative $f_{u}$ exists on $D$ and satisfies $f_{u}(t, u)>0$ on $D$. H-3: $|f(t, u(t))| \leqq f_{u}(t, 0)|u(t)|$ on $D$.

An important class of functions $f(t, u)$ which satisfy conditions $\mathrm{H}-1,2,3$ is the class of twice continuously differentiable functions $f(t, u)$ which are odd and strictly monotone in $u$ with $f_{u u} \geqq 0$ for $u<0$ and $f_{u u} \leqq 0$ for $u>0$. Nonlinear eigenvalue problems involving this class of functions have been studied extensively by G. H. Pimbley [1].

For the case $f(t, u)= \pm t^{\sigma} u^{n}, \mathrm{R}$. Bellman [2] has given an exhaustive treatment of the asymptotic behavior of proper solutions (i.e., solutions which exist and have continuous derivatives for $\left.t \geqq t_{0}\right)$. For the case $f(t, u)=a(t) u^{2 n+1}$ several results on asymptotic behavior exist depending on properties of $a(t)$. References can be found in the papers of P. Waltman [3] and R. A. Moore and Z. Nehari [4].

Our basic result is that there exist solutions of (1) which approach those of $u^{\prime \prime}=0$. More precisely, we prove the

Received by the editors October 5, 1966.

1 This work was supported by the National Science Foundation under Grant No. GP-4597 at the California Institute of Technology. 
ThEOREM. Let $f(t, u)$ satisfy $\mathrm{H}-1,2,3$, and in addition, suppose that

$$
\int^{\infty} t f_{\mu}(t, 0) d t<\infty .
$$

Then, equation (1) has solutions which are asymptotic to $a+b t$ as $t \rightarrow \infty$, where $b \neq 0$.

Proof. Our proof is essentially a modification of one given by Bellman [2, pp. 114-115] for the linear case $f(t, u)=a(t) u$. Integrate (1) twice between 1 and $t$ to obtain

$$
u(t)=c_{1}+c_{2} t-\int_{1}^{t}(t-s) f(s, u(s)) d s .
$$

From this we obtain, for $t \geqq 1$,

$$
|u(t)| \leqq\left(\left|c_{1}\right|+\left|c_{2}\right|\right) t+t \int_{1}^{t}|f(s, u(s))| d s .
$$

Using properties $\mathrm{H}-2,3$, we then obtain

$$
\frac{|u(t)|}{t} \leqq\left(\left|c_{1}\right|+\left|c_{2}\right|\right)+\int_{1}^{t} s f_{u}(s, 0) \frac{|u(s)|}{s} d s .
$$

We now invoke the fundamental Gronwall inequality which states that if $u, v \geqq 0, c>0$ and if $u(x) \leqq c+\int_{0}^{x} u(\xi) v(\xi) d \xi$, then

$$
u(x) \leqq c \exp \left(\int_{0}^{x} v(\xi) d \xi\right)
$$

Applying this to (4), we obtain

$$
\frac{|u(t)|}{t} \leqq\left(\left|c_{1}\right|+\left|c_{2}\right|\right) \exp \left(\int_{1}^{t} s f_{u}(s, 0) d s\right) .
$$

Finally, (2) and (5) imply

$$
|u(t)| / t \leqq c_{3} .
$$

Now, by differentiating (3), we obtain

$$
u^{\prime}(t)=c_{2}-\int_{1}^{t} f(s, u(s)) d s .
$$

Then, H-3 and (6) imply that

$$
\int_{1}^{t}|f(s, u(s))| d s \leqq \int_{1}^{t} f_{u}(s, 0)|u(s)| d s \leqq c_{3} \int_{1}^{t} s f_{u}(s, 0) d s .
$$


Hence, as $t \rightarrow \infty$, the integral in (7) converges, and therefore $u^{\prime}$ has a limit as $t \rightarrow \infty$. To ensure that this limit is not zero, we choose $c_{2}=1$ and use as a lower limit, instead of 1 , a point $t_{0}$, where $t_{0}$ is chosen so that $c_{3} \int_{t_{0}}^{\infty} s f_{u}(s, 0) d s<1$.

Q.E.D.

\section{REFERENCES}

1. G. H. Pimbley, Jr., A sublinear Sturm-Liouville problem, J. Math. Mech. 11 (1962), 121-138.

2. R. Bellman, Stability theory of differential equations, McGraw-Hill, New York, 1953.

3. P. Waltman, On the asymptotic behavior of solutions of a nonlinear equation, Proc. Amer. Math. Soc. 15 (1964), 918-923.

4. R. A. Moore and Z. Nehari, Nonoscillation theorems for a class of nonlinear differential equations, Trans. Amer. Math. Soc. 93 (1959), 30-52.

California Institute of Technology 